\section{Mathematics Is a Quest for Truth}

The question of how to teach mathematics in primary and secondary education has appeared in newspaper opinion pages regularly during the last few years. The topic is a complicated one and does not allow for easy or simple answers. I am most alarmed, however, by proposals to eliminate abstract mathematical reasoning and subjects such as algebra and Euclidean geometry from the curriculum, presenting math as a collection of formulas and algorithms motivated by their applications to the "real world". Instead, I believe that mathematics should be taught because it is a fundamental human endeavor, an essential part of the search for truth and meaning in our lives.

The notion of truth appears to be in short supply today, despite the overflow of information. We are inundated daily through the news media with ambiguous definitions, unstated premises, and questionable theories about human affairs. Science is certainly not immune to this problemone need only look a few decades in the past to see how the prevailing orthodoxy in a given field affects the state of the art. Mathematics stands out among human pursuits because its conclusions enjoy a permanency unmatched in other subjects. The mathematical wisdom of ancient times is just as valid today as it was then, and we ignore it at our own peril. On the other hand, mathematicians are typically humble about their own achievements, because they stand in awe before the vastness of what they do not know.

The word "mathematics" comes from the Greek "mathema", which means "lesson". For the ancient Greeks, one was not considered educated without a thorough grasp of mathematics, and famously could not enter into Plato's academy without knowing geometry. This reflects not only mathematics's connections to philosophical thought, but the uncanny way in which it seems to explain, and indeed to govern, the world. But mathematics is not merely a Platonic realm of our imagination, best left to the few that can climb its intellectual heights. The most important reason to provide abstract mathematics to the general population is to teach people to think critically about the world, to question their assumptions, and to illustrate the power of logical deduction. For mathematics is one of the few areas where the boundary between what is known and what is not known is well defined, and where we can always answer the question "why?" about the former.

Whenever one slices through a carrot or a log of wood along a slanted plane, the border of the oval-shaped cross section is a curve called an ellipse. Surprisingly, the paths that the planets trace as they move around the sun are also described by ellipses. This fact was first observed by Kepler, and proved mathematically by Newton, in one of the great triumphs of scientific thought. Nearly two thousand years of inquiry separate these two very different ways of viewing the same curve. Many examples like this show how abstract mathematical constructions stemming from everyday human experience arise in unexpected places and instill within us a sense of wonder at the inner workings of creation.

The most familiar of all mathematical objects are the numbers we use to count; yet even their genealogy is far from simple. The number zero provides a striking example, because it has a traceable history. The ancient Greeks and Romans lacked it, as was made clear during the turn of the millennium: did it occur in 2000 or in 2001 ? The problem is that there was no year 0 A.D., because there was no Roman numeral zero. Indeed, why does one need a number to count objects which do not exist? The invention (or discovery) of the number zero occurred independently in different parts of the world, but principally in India and Central America-and in both cases, the reason for its introduction was religious. This story indicates that the roots of mathematical thought lie within the deepest recesses of the human mind, where logos and mythos come together, in search of our very nature.

There are many practical problems that arise when trying to get the above message across to students. However, I am encouraged by two personal convictions: I believe that students are capable of much more than we give them credit for, and that they respond positively to challenges, provided that they are suitably motivated. I am also convinced that there is a thirst for mathematical education, that people are inherently fascinated by the profound beauty and power of the subject, and that a knowledgeable, passionate teacher can do wonders with it.

Mathematics has been called the music of reason, and we do our children a grave disservice if we reduce it to a cacophony of disconnected formulas. Indeed, we risk losing what is perhaps the most precious inheritance of all.

-Harry Tamvakis University of Maryland harryt@math.umd.edu 